\title{
AN ALGEBRA OF GENERALIZED FUNCTIONS ON AN OPEN INTERVAL; TWO-SIDED OPERATIONAL CALCULUS
}

\author{
BY GREGERS KRABBE
}

Communicated by Philip Hartman, July 23, 1970

Let $\Omega$ be an open subinterval of the real line; suppose that $0 \in \Omega$. The purpose of this announcement is to describe an injection of $L^{\text {loo }}(\Omega)$ into a commutative algebra of operators. The injection is a useful substitute for the two-sided Laplace transformation; in case $\Omega$ is the whole real line, the injection can be extended to a space $\mathscr{B}$ of distributions whose supports may be all of $(-\infty, \infty)$ (there are no growth restrictions: see $\$ 7$ ). If the distributional derivative of an arbitrary distribution $R$ belongs to the space $\mathfrak{B}$, then $R$ also belongs to $\mathfrak{B}$ and $R$ has an initial value (because $R$ equals a continuous function in some left-neighborhood of the origin). Thus, it is possible to assign initial conditions to the unknown distribution in a differential equation whose right-hand side belongs to the space $\mathfrak{B}$ : see 7.3.

1. Preliminaries. If $f_{1}()$ and $f_{2}()$ belong to the space $L^{\text {loc }}(\Omega)$ (of all the complex-valued functions which are integrable on each compact subinterval of the open set $\Omega$ ), we denote by $f_{1} \wedge f_{2}()$ the function defined by

$$
f_{1} \wedge f_{2}(t)=-\int_{t}^{0} f_{1}(t-u) f_{2}(u) d u
$$

(for all $t$ in $\Omega$ ).

2. The space of test-functions. Let $W \Omega$ be the space of all the complex-valued functions which are infinitely differentiable on $\Omega$ and whose every derivative vanishes at the origin. Thus, $w()$ belongs to $W \Omega$ if $w() \in C^{\infty}(\Omega)$ and $w^{(k)}(0)=0$ for every integer $k \geqq 0$.

2.1. The space of generalized functions. Let $Q \Omega$ be the space of all the linear operators $A$ which map $W \Omega$ into $W \Omega$ such that the equation

AMS 1970 subject classifications. Primary 44A40, 44A35, 42A76; Secondary 46F99, 34A10, 45A05.

Key words and phrases. Generalized functions, operational calculus, direct sums of algebras, Schwartz distributions, initial-value problems, two-sided Laplace transformation, Fourier transformation, Mikusinski operational calculus, operateurs de Heaviside, translation properties, initial values, Dirac distributions, distributional derivative. 


$$
A\left(w_{1} \wedge w_{2}\right)(t)=\left(A w_{1}\right) \wedge w_{2}(t) \quad(\text { for all } t \text { in } \Omega)
$$

holds whenever $w_{1}()$ and $w_{2}($ ) belong to $W \Omega$. As usual, if $w($ ) belongs to $W \Omega$, then $A w()$ denotes the image of $w()$ under the operator A.

2.2. The injection. If $f()$ belongs to $L^{\text {loc }}(\Omega)$ we denote by $\{f(t)\}$ the operator $w() \mapsto(f \wedge w)^{\prime}()$ which assigns to each $w()$ in $W \Omega$ the derivative of the function $f \wedge w()$.

THEOREM. The linear transformation $f() \mapsto\{f(t)\}$ is one-to-one and maps $L^{\text {loc }}(\Omega)$ into the linear space a $\Omega$.

2.3. The operational calculus. The linear space $Q \Omega$ is a commutative algebra with unit element 1 (the identity operator), multiplication being the usual composition of operators.

If $f(t)=1$ for $t \in \Omega$, then $\{f(t)\}$ is the identity operator 1 (defined by $1 w()=w()$ for all $w()$ in $W \Omega$ ). The differentiation operator $D$ (defined by $D w()=w^{\prime}()$ for all $w()$ in $W \Omega$ ) is an invertible element of the algebra $Q \Omega$ such that the equation

$$
\left\{f_{1} \wedge f_{2}(t)\right\}=\left\{f_{1}(t)\right\} D^{-1}\left\{f_{2}(t)\right\}
$$

holds for every $f_{1}()$ and $f_{2}()$ in $L^{\text {loo }}(\Omega)$. The preceding properties imply all the usual operational formulas. For example, if $f()$ is locally absolutely continuous on $\Omega$, then

$$
\left\{f^{\prime}(t)\right\}=D\{f(t)\}-f(0) D
$$

whence

$$
\{\sin t\}=\frac{D}{D^{2}+1} \text { and }\{\cos t\}=D\{\sin t\}
$$

We can now solve problems such as

$$
y^{\prime \prime}(t)+y(t)=\sec (\pi t / 2 \omega) \quad(-\omega<t<\omega)
$$

to that effect, set $\Omega=(-\omega, \omega), c_{0}=y(0), c_{1}=y^{\prime}(0)$, and inject both sides of (1) into $a \Omega$; from (2.5) it follows that

$$
\{y(t)\}=c_{0} D \frac{D}{D^{2}+1}+c_{1} \frac{D}{D^{2}+1}+\frac{D}{D^{2}+1} D^{-1}\left\{\sec \frac{\pi t}{2 \omega}\right\} ;
$$

from (2.4), (2.6), and (1.1) it therefore results that

$$
y(t)=c_{0} \cos t+c_{1} \sin t-\int_{t}^{0}[\sin (t-u)] \sec \frac{\pi u}{2 \omega} d u .
$$

2.7. Other operational calculi. Mikusinski's injection (of $L^{100}(0, \infty)$ 
into the Mikusinski field) is an extension of the Laplace transformation; analogously, our injection $f() \mapsto\{f(t)\}$ can be compared with the two-sided Laplace transformation. However, the two-sided Laplace transforms

$$
\mathfrak{R}\left\{e^{-t}-e^{t}\right\} \text { and } \mathfrak{R}\left\{e^{-|t|}\right\}
$$

are restrictions of the same function to different vertical strips; contrastingly,

$$
\left\{e^{-t}-e^{t}\right\}=\frac{2 D}{1-D^{2}} \text { and }\left\{e^{-|t|}\right\}=\frac{D^{2}-D \Sigma}{D^{2}-1}
$$

where $\Sigma=\{\operatorname{sgn} t\}=\{t /|t|\}$. More generally, if $-\infty<\lambda<\infty$ then

$$
\left\{e^{\lambda|t|}\right\}=\frac{D^{2}+\lambda D \Sigma}{D^{2}-1}
$$

A problem which is not Laplace transformable is discussed in 7.8.

3. Direct sum decomposition. Let $1_{+}()$be the Heaviside step function; we set $1_{+}=\left\{1_{+}(t)\right\}, 1_{-}=1-1_{+}$, and

$$
(B Q)=\{B A: A \in a \Omega\} \quad \text { (when } B \in Q \Omega \text { ). }
$$

Both $\left(1_{-} Q\right)$ and $\left(1_{+} Q\right)$ are ideals in the algebra $Q \Omega$, and their direct sum equals $Q \Omega$ :

$$
a \Omega=(1-a) \oplus\left(1_{+} a\right) .
$$

In fact, $1_{-}$(respectively, $1_{+}$) is a projector of $Q \Omega$ into (1_Q) (respectively, $\left.\left(1_{+} Q\right)\right),\left(1_{-}\right)^{2}=1=\left(1_{+}\right)^{2}$, and $\left(1_{-}\right)\left(1_{+}\right)=0$.

3.1. REMARK. If $h() \in L^{\text {loc }}(\Omega)$ then $\left\{h_{-}(t)\right\} \in\left(1_{-} Q\right)$ and $\left\{h_{+}(t)\right\}$ $\in\left(1_{+} Q\right)$, where $h_{+}()=1_{+}() h()$ and

$$
h_{-}()=h()-h_{+}() \text {. }
$$

4. Translation properties. If $-\infty<x<\infty$ we define the function $1^{x}()$ by $1^{x}(t)=0$ for $-|x| \leqq t<|x|$, and by $1^{x}(t)=1$ for all other values of $t$. Further, we set $1^{x}=\left\{1^{x}(t)\right\}$.

4.1. Theorem. Suppose that $\alpha>0$ and $\lambda \geqq 0$; if $h() \in L^{100}(\Omega)$ then the equation

$$
\frac{1^{\lambda}\{h(t)\}}{1-c 1^{\alpha}}=\left\{\sum_{k=0}^{\infty} c^{k}\left[h_{-}(t+k \alpha+\lambda)+h_{+}(t-k \alpha-\lambda)\right]\right\}
$$

holds for any complex number $c$. 
4.3. Comments. The series inside the right-hand bracket is a locally finite sum. Theorem 4.1 is the two-sided extension of Theorem 5.29 in [4]. If $h()$ is a periodic function having period $\alpha>0$, then

$$
\{h(t)\}=\frac{\left\{\left[1-1^{\alpha}(l)\right] h(t)\right\}}{1-1^{\alpha}} .
$$

5. The topology. Let the function-space $W \Omega$ be equipped with the topology of pointwise convergence on the interval $\Omega$; since $Q \Omega$ consists of mappings of $W \Omega$ into the topological space $W \Omega$, we can equip a $\Omega$ with the product topology. The following results have been proved by Harris Shultz: the topology of the linear space $a \Omega$ is sequentially complete, locally convex, and Hausdorff; moreover, the multiplication of the algebra $a \Omega$ is sequentially continuous.

5.1. The translation operator. If $-\infty<x<\infty$ we set $\mathbf{T}_{x}=\left\{\mathrm{T}_{x}(t)\right\}$, where

$$
T_{x}()=-1_{-}(x) 1_{-}^{x}()+1_{+}(x) 1_{+}^{x}() .
$$

It turns out that $D \mathrm{~T}_{x}=\lim \left\{\epsilon^{-1} F_{e}(t)\right\}$ (as $\left.\epsilon \rightarrow 0+\right)$, where $F_{e}()$ is the characteristic function of the interval $(x, x+\epsilon)$; this indicates that $D \mathrm{~T}_{x}$ corresponds to the Dirac distribution $\delta_{x}$ concentrated at the point $x$. If $h() \in L^{100}(\Omega)$ is a periodic function of period $\alpha>0$, then the equation

$$
\{h(t)\} \sum_{k=-\infty}^{\infty} c_{k} \mathrm{~T}_{k \alpha}=\left\{h(t) \sum_{k=-\infty}^{\infty} c_{k} \mathrm{~T}_{k \alpha}(t)\right\}
$$

holds for any complex-valued sequence $c_{k}(k=0, \pm 1, \pm 2, \pm 3, \cdots)$.

6. Initial values. Given $A$ and $B$ in $Q \Omega$, we shall say that $A$ equals $B$ on an interval if $A w(t)=B w(t)$ for all $t$ in the interval and for all $w()$ in $W(-\infty, \infty)$. For example, any element of $(1+a)$ equals 0 on $(-\infty, 0)$.

6.1. Definition. A number $c$ is called an initial value (of $B$ ) if $c=\lim f(t)$ (as $t \rightarrow 0-$ ) for some function $f()$ such that $\{f(t)\}$ equals $B$ on some interval $(\lambda, 0)$.

6.2. REMARKs. If the set of initial values (of $B$ ) is not void, it contains a unique element $B(0-)$; we shall see in 7.3 that the operator $D B-B(0-) D$ corresponds to the distributional derivative.

7. A new space of distributions. Let $\mathfrak{B}_{L}$ (respectively, $\mathfrak{B}_{+}$) be the space of all the Schwartz distributions whose supports are contained in the half-open interval $(-\infty, 0]$ (respectively, $[0, \infty)$ ). Let $\mathfrak{B}_{-}$be 
the space of all the elements of $\mathfrak{B}_{L}$ that are regular in some open neighborhood of the origin; we set

$$
\mathfrak{B}=\mathfrak{B}_{-}+\mathfrak{B}_{+} \text {. }
$$

Thus, $\mathfrak{B}$ is the family of all the sums $S+R$, where $R$ is a distribution with Supp $R \subset[0, \infty)$, and where $S \in \mathscr{B}_{-}$(that is, Supp $S$ is contained in $(-\infty, 0]$ and there exists a distribution $\partial^{\circ} f$ corresponding to a function $f()$ such that $S-\partial^{0} f$ is zero in some open interval containing the origin). It turns out that $\mathfrak{B}$ is the direct sum $\mathfrak{B}_{-} \oplus \mathfrak{B}_{+}:$if $F \in \mathfrak{B}$ there exists a unique pair $\left(F_{-}, F_{+}\right)$in $\mathfrak{B}_{-} \times \mathfrak{B}_{+}$such that $F=F_{-}+F_{+}$. If $F \in \mathfrak{B}$ and $G \in \mathfrak{B}$ we set

$$
F \otimes G=-F_{-} \star G_{-}+F_{+} \star G_{+},
$$

where $\star$ is convolution in the sense of $[3$, p. 384]. By adjoining the multiplication $(F, G) \mapsto F \otimes G$ to the linear space $\mathfrak{B}$ we obtain a commutative ring. Denoting by $\partial^{0} f$ the distribution corresponding to a function $f()$, we have $\left(\partial^{0} f_{1}\right) \otimes\left(\partial^{0} f_{2}\right)=\partial^{0}\left(f_{1} \wedge f_{2}\right)$.

If $F \in \mathfrak{B}$ and $w() \in W(-\infty, \infty)$ then $F \otimes\left(\partial^{0} w^{\prime}\right)$ is the distribution corresponding to a function in $W(-\infty, \infty)$ that we shall denote by $\{F\} w()$; it turns out that $\{F\} w()$ belongs to $W(-\infty, \infty)$. Let $\{F\}$ denote the mapping $w() \rightarrow\{F\} w()$ (of $W(-\infty, \infty)$ into itself); if $\delta_{x}$ is the Dirac distribution, then

$$
\left\{\delta_{x}\right\}=D \mathrm{~T}_{x} \quad(-\infty<x<\infty) .
$$

7.2. THEOREM. The transformation $F \mapsto\{F\}$ is a linear injection of $\mathfrak{B}$ into $a(-\infty, \infty)$ such that $\left\{\partial^{\circ} f\right\}=\{f(t)\}$ for $f() \in L^{l o c}(-\infty, \infty)$ and

$$
\left\{F_{1} \otimes F_{2}\right\}=\left\{F_{1}\right\} D^{-1}\left\{F_{2}\right\} \quad\left(\text { for } F_{1} \text { and } F_{2} \text { in } \mathfrak{B}\right) \text {. }
$$

7.3. THEOREM. If $R$ is a distribution whose distributional derivative belongs to $\mathfrak{B}$, then the set of initial values (of $\{R\}$ : see 6.1) contains a unique element which we shall denote by $\langle R, 0-\rangle$; further,

$$
\{\partial R\}=D\{R\}-\langle R, 0-\rangle D \text {. }
$$

as usual, $\partial R$ denotes the distributional derivative of $R$.

7.4. Differential equations. Given $S$ in $\mathfrak{B}$, and let $a_{k}(k=0,1$, $2, \cdots, n$ ) be a set of at least two complex numbers; if $y$ is a distribution such that

$$
\left(a_{n} \partial^{n}+\cdots+a_{1} \partial+a_{0}\right) y=S,
$$

then the distributional derivative $\partial^{k} y$ belongs to $\mathfrak{B}$ for $0 \leqq k \leqq n$; from 
7.3 it therefore follows that we can take in to account the initial values $\left\langle\partial_{y}^{\nu}, 0-\right\rangle$ when $0 \leqq \nu<n$. The equation (7.5) implies that

$$
\left\{\partial^{k} y\right\}=D^{k}\{y\}-\sum_{\nu=0}^{k-1}\left\langle\partial^{\nu} y, 0-\right\rangle D^{k-\nu} \quad(\text { for } 0 \leqq k \leqq n) .
$$

7.7. THEOREM. If $c_{k}(k=0,1,2, \cdots, n-1)$ is a sequence of complex numbers, there exists one and only one distribution y satisfying (7.5) and the initial conditions

$$
\langle y, 0-\rangle=c_{0}, \quad\langle\partial y, 0-\rangle=c_{1}, \cdots, \quad\left\langle\partial^{n-1} y, 0-\right\rangle=c_{n-1} .
$$

7.8. An example. The distributional equation

$$
\partial^{2} y+y=\sum_{k=-\infty}^{\infty} \delta_{2 k \pi}
$$

(discussed on p. 128 of [1]) cannot be solved by the method of fundamental (or "elementary") solutions-nor can it be solved by using the finite Fourier transform [5, pp. 335-342]. However, it can readily be solved by injecting it into $Q(-\infty, \infty)$ : from (1), (7.6), and (7.1) it follows that

$$
\left(D^{2}+1\right)\{y\}=c_{0} D^{2}+c_{1} D+\sum_{k=-\infty}^{\infty} D \mathrm{~T}_{2 k \pi},
$$

where $c_{0}=\langle y, 0-\rangle$ and $c_{1}=\langle\partial y, 0-\rangle$. A particular solution results by setting $c_{0}=c_{1}=0$; solving (2) for $\{y\}$, we can use (2.6) to obtain

$$
\{y\}=\frac{1}{D^{2}+1} D \sum_{k=-\infty}^{\infty} \mathrm{T}_{2 k \pi}=\{\sin t\} \sum_{k=-\infty}^{\infty} \mathrm{T}_{2 k \pi} ;
$$

from (5.2) it now follows the answer

$$
y(t)=\sin t \sum_{k=-\infty}^{\infty} \mathrm{T}_{2 k \pi}(t)=\left(1+\left[\frac{t}{2 \pi}\right]\right) \sin t
$$

$(-\infty<t<\infty)$; as usual, $[t / 2 \pi]$ is the greatest integer $<t / 2 \pi$. The answer (3) cannot be obtained by Fourier transform methods.

7.9. Acknowledgments. At the origin of Theorem 7.7 is an article by César de Freitas [2]; his "opérateurs de Heaviside" are linear combinations of functions with distributions of finite order whose supports are locally finite; these distributions form a proper subspace of our space $\mathfrak{B}$. Harris Shultz gave me the idea that $F \otimes G \in \mathfrak{B}$ whenever both $F \in \mathfrak{B}$ and $G \in \mathfrak{B}$. 


\section{REFERENCES}

1. H. Bremermann, Distributions, complex variables, and Fourier transforms, Addison-Wesley, Reading, Mass., 1965. MR 34 \#8174.

2. A. César de Freitas, Sur les distributions qui interviennent dans le calcul symbolique des electrotechniciens (cas des circuits a constantes concentrés), Univ. Lisboa. Revista Fac. Ci. A. (2) 3 (1954/55), 279-310. MR 17, 613.

3. J. Horváth, Topological vector spaces and distributions, Vol. 1, Addison-Wesley, Reading, Mass., 1966. MR 34 \#4863.

4. G. Krabbe, Operational calculus, Springer-Verlag, Berlin, 1970.

5. A. H. Zemanian, Distribution theory and transform analysis. An introduction to generalized functions, with applications, McGraw-Hill, New York, 1965. MR 31 \#1556.

Purdue University, Lafayette, Indiana 47907 